\title{
Anabases
}

ANABASES Traditions et réceptions de l'Antiquité

12 | 2010

Varia

\section{Jeffrey S. RUSTEN, Thucydides. Oxford Readings in} Classical Studies

Pascal Payen

\section{(2)enEdition}

Journals

Édition électronique

URL : http://journals.openedition.org/anabases/2072

DOI : 10.4000/anabases. 2072

ISSN : 2256-9421

Éditeur

E.R.A.S.M.E.

Édition imprimée

Date de publication : 1 octobre 2010

Pagination : 259-261

ISSN : 1774-4296

Référence électronique

Pascal Payen, « Jeffrey S. Rusten, Thucydides. Oxford Readings in Classical Studies », Anabases [En ligne], 12 | 2010, mis en ligne le 01 juillet 2011, consulté le 22 septembre 2020. URL : http://

journals.openedition.org/anabases/2072 ; DOI : https://doi.org/10.4000/anabases.2072

Ce document a été généré automatiquement le 22 septembre 2020.

(c) Anabases 


\title{
Jeffrey S. RUSTEN, Thucydides. Oxford Readings in Classical Studies
}

\author{
Pascal Payen
}

\section{RÉFÉRENCE}

Jeffrey S. RUSTEN, Thucydides. Oxford Readings in Classical Studies, Oxford, Oxford

University Press, 2009, x-519 p.

35 livres / ISBN 978-0-19-920619-3.

1 Le livre édité par Jeffrey Rusten est bien plus qu'un recueil d'études consacrées à Thucydide. Tel qu'il est conçu et par la mise en perspective d'ensemble qu'il propose, il s'agit d'un remarquable instrument de travail, indispensable aussi bien aux lecteurs qui découvrent Thucydide qu'aux spécialistes de son œuvre.

Le livre réunit dix-neuf contributions empruntant à toutes les "écoles", depuis un chapitre de J. de Romilly consacré aux récits de bataille dans Histoire et raison chez Thucydide (1956) jusqu'à une analyse de J. Ober (2001), tirée d'un livre où l'historien athénien est sollicité par les sciences politiques contemporaines comme modèle «théorique » permettant de rapprocher la guerre du Péloponnèse et la guerre de Corée. Dans ce livre, l'accent est mis délibérément non sur la guerre du Péloponnèse elle-même en tant qu'objet historique, mais sur Thucydide historien et écrivain de l'événement. La construction du livre reflète ce choix. La partie introductive comporte quatre contributions (de W. R. Connor, K. J. Dover, S. Hornblower, précédées de l'étude liminaire de l'éditeur) qui reflètent bien les débats interprétatifs autour du grand écart entre le Thucydide des historiens et celui des littéraires, et des tentations "postmodernistes " des années 1970. Les douze études qui suivent, toutes traduites en anglais (le tiers d'entre elles pour la première fois), sont des analyses approfondies, qui ont fait date, de passages particuliers tirés des livres I, II, III, V, VI, VII. On retrouvera les contributions de L. Edmunds, C. Dewald, T. Rood, Chr. Pelling, H. Strasburger, J. Vogt, B. Manuwald, N. Loraux, H. D. Westlake, B. Bosworth, H.-P. Stahl, J. de Romilly. 
Une dernière section aborde des questions de réception (R. Nicolai pour l'Antiquité ; J. Scott et la guerre civile en Angleterre, entre 1618 et 1648 ; J. Ober ,déjà signalé). On s'étonnera toutefois de ne voir aucune contribution consacrée aux livres IV et VIII. Pourquoi n'avoir pas retenu, par exemple, l'une des études que D. Babut a livrées au sujet du livre IV : "Interprétation historique et structure littéraire chez Thucydide: remarques sur la composition du livre IV » (Bulletin de l'Association Guillaume Budé, 4, 1981, repris in Parerga. Choix d'articles (1974-1994), Lyon, Maison de l'Orient méditerranéen, 1994, p 585-607) ou «L'épisode de Pylos-Sphactérie chez Thucydide : l'agencement du récit et les intentions de l'historien » (Revue de philologie, 60, 1986, repris in Parerga, p. 641-661), études qui ne figurent pas non plus dans la section de la bibliographie réservée au livre IV, p.506-507. Il est vrai que l'éditeur et l'auteur précisent que sont privilégiés les travaux de langue anglaise.

Mais le grand apport et l'utilité de ce livre proviennent de l'organisation que lui a conférée son auteur et dont il s'explique dans l'étude liminaire : "Thucydides and his readers" (p.1-28). J. Rusten a mis en rapport son introduction avec la riche bibliographie finale, de 435 références, numérotées et classées en 36 rubriques, qui constituent autant d'entrées d'une sorte de "dictionnaire - Thucydide» et dont l'originalité dans les choix offre, à côté de titres attendus et indispensables ("Bibliographical surveys", "Text and scholia», "Specific words and concepts", «Internal politics »...), des perspectives de lectures neuves qu'il est possible de croiser, ainsi des titres suivants: "Traditionalism, emotionalism, and pathos", "Intellectual milieu », « Authorial persona », « Ancient reception », « Later reception ». Le lecteur est donc invité à poursuivre ses recherches bien au-delà des contributions du présent livre, et J. Rusten lui en donne les moyens par la mise en perspective de ses propres analyses, qui réintroduisent le lecteur dans l'œuvre de Thucydide alors que ce dernier s'est savamment ingénié à lui refuser, on le sait, toute place. Thucydide a construit un monument - «la guerre des Péloponnésiens et des Athéniens », telle que lui-même l'a mise par écrit (xunegrapsé) - que le lecteur est sommé d'admettre comme tel. Thucydide, en effet, a exploré toutes les causes de la guerre "afin d'éviter que personne (mè tina) ne se demande un jour d'où sortit, en Grèce, une guerre pareille » (I, $23,5)$ : ce " personne » est l'unique mention où le lecteur a droit de cité, encore est-ce sur le mode négatif.

Pour que le lecteur moderne, à défaut de concurrencer l'historien ancien, circule dans l'œuvre, J. Rusten propose trois parcours. Tout d'abord, il est possible de suivre un ordre chronologique, celui de ce qu'il est convenu d'appeler la « réception » de l'œuvre, depuis les premiers lecteurs et critiques, Platon et Isocrate. Les pages 25 à 28 dressent ainsi un utile classement et répertoire (forcément incomplet) des grands lecteurs de Thucydide et des moments les plus significatifs qui leur font suite : la Rome de Cicéron et de Dion Cassius (mais Byzance est ensuite oubliée) ; la Renaissance de Leonardo Bruni, Lorenzo Valla et Alde Manuce; les temps modernes, de Hobbes à Roscher (et Pierre-Charles Lévêque, 1795); la première grande remise en cause avec Cornford (1907) ; puis les « Post-WWI Studies» de Schwartz et Thibaudet; le temps des «Antiseparatist Studies »; les « Post-WWI Studies » de J. de Romilly à Strasburger, avant que ne s'imposent, pour plusieurs décennies, le positivisme critique initié par le commentaire de Gomme. Celui-ci ne pouvait que favoriser en retour les approches tantôt « littéraires » tantôt déconstructionnistes de Stahl, Hunter, Loraux, Connor. Il est banal de dire que les questions posées à Thucydide sont celles du présent de celui qui le lit; les recherches des départements de sciences politiques, aux États-Unis et 
ailleurs - dernière étape de ce parcours chronologique -, en témoignent. Le second parcours proposé dans les études thucydidéennes est thématique ou par grands sujets (p. 3-15) : la question de l'unité de l'œuvre, depuis la thèse d'Ullrich (1846), pour qui le projet initial de Thucydide se serait arrêté en 421 (V, 24); les discours; l'historiographie et l'art $\mathrm{du}$ récit; la question de l'impérialisme et des relations internationales; enfin trois approches actuelles - "narratology ", " intertextuality ", « reception "-, qui auraient dû être distinguées tant la dernière ne recoupe pas les deux premières. Le troisième parcours propose une lecture "livre par livre", section par section (p.15-17). Pour permettre au lecteur de s'approprier pleinement cet instrument de travail, l'auteur, dans la dernière section (p. 17-24), résume de façon concise et nette chacune des dix-neuf contributions.

5 L'ensemble témoigne d'une grande connaissance des études sur Thucydide, dans le temps long. La précision des synthèses et des présentations, l'efficacité des renvois permettent au lecteur, étudiant ou spécialiste de ces problèmes, de circuler dans l'immense bibliographie consacrée à Thucydide, dont il faut redire qu'il fut considéré, dans l'université et la société allemandes du xixe siècle, comme un des modèles de l'histoire alors en train de se constituer comme science et comme discipline. Pour ces raisons, un tel livre doit être mis entre les mains de tous les historiens.

\section{AUTEURS}

PASCAL PAYEN

Université de Toulouse (UTM)

payen@univ-tlse2.fr 